ARTICLE

Received 25 Mar 2013 | Accepted 24 Nov 2013 | Published 8 Jan $2014 \quad$ DOl: 10.1038/ncomms4.005

\title{
Ultra-high mobility transparent organic thin film transistors grown by an off-centre spin-coating method
}

Yongbo Yuan', Gaurav Giri², Alexander L. Ayzner ${ }^{2,3}$, Arjan P. Zoombelt ${ }^{2}$, Stefan C. B. Mannsfeld ${ }^{3}$, Jihua Chen ${ }^{4}$, Dennis Nordlund ${ }^{3}$, Michael F. Toney ${ }^{3}$, Jinsong Huang ${ }^{1} \&$ Zhenan Bao ${ }^{2}$

Organic semiconductors with higher carrier mobility and better transparency have been actively pursued for numerous applications, such as flat-panel display backplane and sensor arrays. The carrier mobility is an important figure of merit and is sensitively influenced by the crystallinity and the molecular arrangement in a crystal lattice. Here we describe the growth of a highly aligned meta-stable structure of 2,7-dioctyl[1]benzothieno[3,2-b][1]benzothiophene (C8-BTBT) from a blended solution of C8-BTBT and polystyrene by using a novel offcentre spin-coating method. Combined with a vertical phase separation of the blend, the highly aligned, meta-stable C8-BTBT films provide a significantly increased thin film transistor hole mobility up to $43 \mathrm{~cm}^{2} \mathrm{Vs}^{-1}\left(25 \mathrm{~cm}^{2} \mathrm{Vs}^{-1}\right.$ on average), which is the highest value reported to date for all organic molecules. The resulting transistors show high transparency of $>90 \%$ over the visible spectrum, indicating their potential for transparent, high-performance organic electronics.

\footnotetext{
${ }^{1}$ Department of Mechanical and Materials Engineering and Nebraska Center for Materials and Nanoscience, University of Nebraska-Lincoln, Lincoln, Nebraska 68588-0656, USA. ${ }^{2}$ Department of Chemical Engineering, Stanford University, Stanford, California 94305, USA. ${ }^{3}$ Stanford Synchrotron Radiation Lightsource, SLAC National Accelerator Laboratory, Menlo Park, California 94025, USA. ${ }^{4}$ Center for Nanophase Materials Sciences, Oak Ridge National Laboratory, Oak Ridge, Tennessee 37831-6494, USA. Correspondence and requests for materials should be addressed to J.H. (email: jhuang2@unl.edu) or to Z.B. (email: zbao@stanford.edu).
} 
T ransparent organic semiconductors with high chargecarrier mobilities have been an important research target due to their broad applications in flat-panel displays ${ }^{1,2}$, radio-frequency identification tags ${ }^{3,4}$, complementary integrated circuits $^{5-7}$ and biological and medical applications ${ }^{8-13}$. The carrier mobility of organic semiconductor films is strongly influenced by the crystallinity, molecular packing structures of the organic thin films and charge traps at the gate dielectric/ semiconductor interface ${ }^{14-17}$. Because of the small van der Waals interaction between organic molecules, the crystallinity, grain size and crystal alignment of the solution-processed organic thin films have been shown to be very sensitive to the fabrication conditions, such as solvent evaporation rate ${ }^{18}$, and liquid surface tension force ${ }^{19,20}$. In addition to the changed thin film morphology, certain molecular organic semiconductors can form various molecular packing structures (polymorphs) by changing film formation processes ${ }^{19,20}$. Since the electronic wavefunction overlap that determines the charge transfer integral is a very sensitive function of the precise molecular packing, the various polymorphs generally have different carrier mobilities with some having a higher mobility than their equilibrium structures ${ }^{10,19-23}$.

In this manuscript, we report the formation of a highly aligned, meta-stable crystal packing structure (likely a polymorph) of 2,7-dioctyl[1]benzothieno[3,2-b][1]benzothiophene (C8-BTBT by a simple off-centre spin-coating (OCSC) method, wherein C8-BTBT and polystyrene (PS) blend solution was used to result in simultaneously a passivation effect of the dielectric surface as well as improving the continuity of the thin C8-BTBT film. An ultra-high maximum hole mobility of $43 \mathrm{~cm}^{2} \mathrm{Vs}^{-1}$ and an average hole mobility of $25 \mathrm{~cm}^{2} \mathrm{Vs}^{-1}$ were obtained, much higher than the previously reported average hole mobility of $3 \sim 16 \mathrm{~cm}^{2} \mathrm{Vs}^{-1}$ (refs 18,24-26).

\section{Results}

OCSC and device transparency. The organic thin film transistors (OTFTs) were fabricated on either highly doped silicon substrates or transparent indium tin oxide (ITO) coated glass substrates with a bottom-gate top-contact structure (Fig. 1a). A crosslinked poly(4-vinylphenol) (PVP) was used as the gate dielectric layer (Supplementary Fig. 1) 27 $^{27}$ The semiconductor channel layer was deposited by an OCSC method, in which the substrate is placed away from the centre of the spin-coater (Fig. $1 \mathrm{~b}$ and Supplementary Fig. 2), using a blend of C8-BTBT with an insulating PS. Since all the organic materials used here have large bandgaps, the resulting films when cast on ITO substrates showed an excellent transparency of $>90 \%$ in the visible region (Fig. 1c), which is comparable to the best transparencies reported to date for TFTs, including both organic and metal oxide TFTs ${ }^{28,29}$. Such highly transparent transistors are of great interest for flat-panel display backplane and sensor array applications.

Formation of highly aligned meta-stable C8-BTBT films. In the OCSC process, the centrifugal force is almost unidirectional over the whole substrate. This method produced highly aligned C8BTBT crystals, as confirmed by their distinct anisotropic optical absorption spectra under varying polarization of incident light. The peak absorbance of the C8-BTBT film formed by the OCSC method is 2.5 times stronger when the light polarization direction is perpendicular to the radial direction as compared with the radial direction (Fig. 1d). In contrast, the C8-BTBT films fabricated by conventional on-centre spin-coating (i.e., substrate centre sitting at the centre of spin-coater axis) showed nearly isotropic light absorption, indicating that crystals in the film were randomly oriented in the plane of the substrate (Fig. 1e). There was a small spectral shift of $\sim 50 \mathrm{meV}$ between the peak positions of the first absorption band for the two different polarizations in the OCSC film (Fig. 1d), which corresponds to the Davydov splitting of the lowest energy transition in the isolated molecule induced by the anisotropic crystal environment. The C8-BTBT crystals formed by the OCSC method all have a small but distinct blue shift of $\sim 20 \mathrm{meV}$ in the absorption spectrum onset as compared with C8-BTBT crystals prepared through other methods, such as small-angle drop-coating (Fig. 1d and Supplementary Methods $)^{18,30}$. The spectral differences are indicative of a change in the crystal packing of C8-BTBT.

To test whether the OCSC film has a meta-stable crystal structure, we measured absorption spectra before and after solvent vapour annealing (o-dichlorobenzene, DCB, Fig. 1f) and thermal annealing $\left(90^{\circ} \mathrm{C}\right.$ for $3 \mathrm{~h}$, Fig. $\left.1 \mathrm{~g}\right)$. Both processes resulted in a redshift of the absorption onset, and the shifted spectra become similar to the spectra obtained from small-angle drop-cast films (Supplementary Methods). In addition, we observed that the spectra of the OCSC films were unchanged after being stored at room temperature for more than 1 month (Supplementary Fig. 3) or being annealed at temperatures below $80^{\circ} \mathrm{C}$ for $3 \mathrm{~h}$, indicating a long lifetime for the meta-stable film (Fig. 1g). Interestingly, it was also noticed that the preferred growth direction of the OCSC film is along the (010) direction of the C8-BTBT crystal (Supplementary Note 1 and Supplementary Fig. 4), which is different from what previously reported, i.e., (100) or (110) directions being the preferred growth direction ${ }^{24,25}$.

To further support the meta-stable phase and the high crystallinity of C8-BTBT, we performed two-dimensional (2D) grazing incidence X-ray diffraction (GIXD) experiments. The observation of an 18th order out-of-plane (11L) Bragg peak from the OCSC processed thin film $(10-20 \mathrm{~nm})$ strongly indicated the highly crystalline nature of our films (Fig. 2a) ${ }^{18}$. The in-plane coherence length by Scherrer analysis provided a lower bound crystallite size of $\sim 100 \mathrm{~nm}$. This crystal coherence length is a lower bound value, as sample degradation and peak broadening occurred with X-ray beam exposure (Supplementary Fig. 5). In contrast, the Bragg peaks of the on-centre spin-coated C8-BTBT films were generally broader, with peak widths corresponding to smaller crystallite sizes $(<20 \mathrm{~nm})$. Moreover, in the on-centre spin-coated films, GIXD intensities on the right and left sides of the image are identical, confirming that the crystallites form a $2 \mathrm{D}$ powder in the plane of the substrate. In contrast, the observed asymmetric pattern in the OCSC film (Supplementary Fig. 6 and Supplementary Note 2) is an indication of the strong in-plane alignment, and is consistent with the optical absorption spectra. Strong evidence for a new crystal packing structure is derived from the presence of an additional diffraction peak near the (002) Bragg reflection, where the lower $Q_{z} \sim 0.44 \AA^{-1}$ is in agreement with the (002) plane spacing reported for the equilibrium crystal $^{31}$, while the higher $Q_{z} \sim 0.46 \AA^{-1}$ is strong evidence for a new polymorph (Fig. 2b). This higher $Q_{z}$ peak is from a metastable phase since it disappears, or decreases in intensity, after thermal annealing (Fig. 2b and Supplementary Fig. 7). In addition, the clear (11L) Bragg peaks shift in position after thermal annealing (Fig. 2c) from $Q_{x y}=1.34 \AA^{-1}$ (meta-stable) to $1.32 \AA^{-1}$ (equilibrium), indicating a smaller intermolecular spacing along (110) direction in the meta-stable phase. The peak shift has previously been observed for other meta-stable systems as well ${ }^{19}$. The meta-stable $(002) \operatorname{Bragg}\left(Q_{z} \sim 0.46 \AA^{-1}\right)$ peak is also present in the on-centre spin-coated samples; however, the crystallite size is too small to give high hole carrier mobilities. Unfortunately, we are unable to obtain the crystal structure for these meta-stable films due to beam degradation of the sample upon long X-ray exposure times required to get accurate meta-stable peak positions and intensities (Supplementary Fig. 5). 


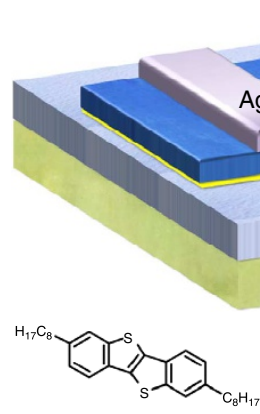

C8-BTBT

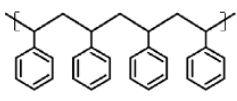

PS

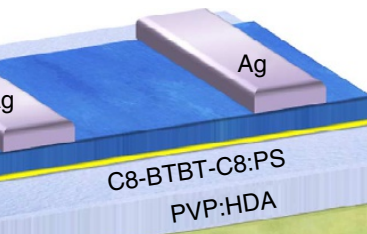

ITO

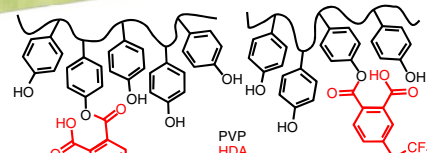

PVP
HDA

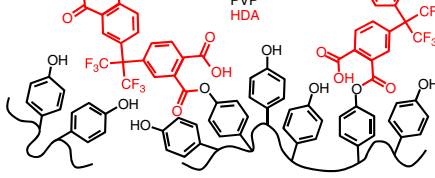

PVP:HDA

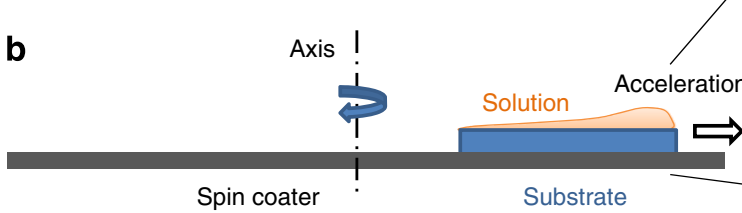

Spin coater

Substrate

e

d

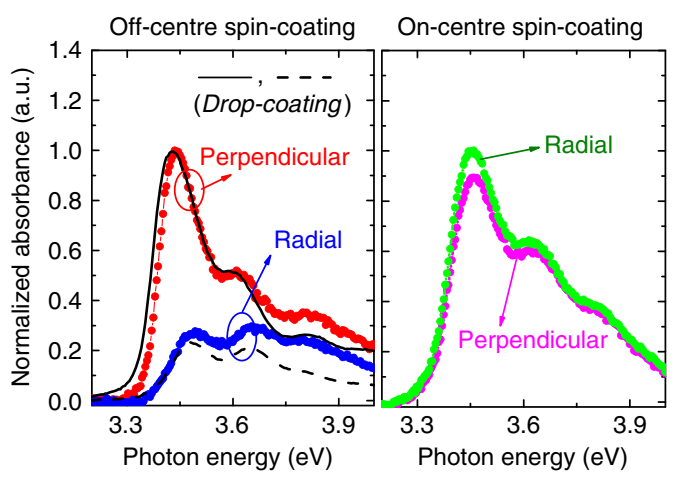

C
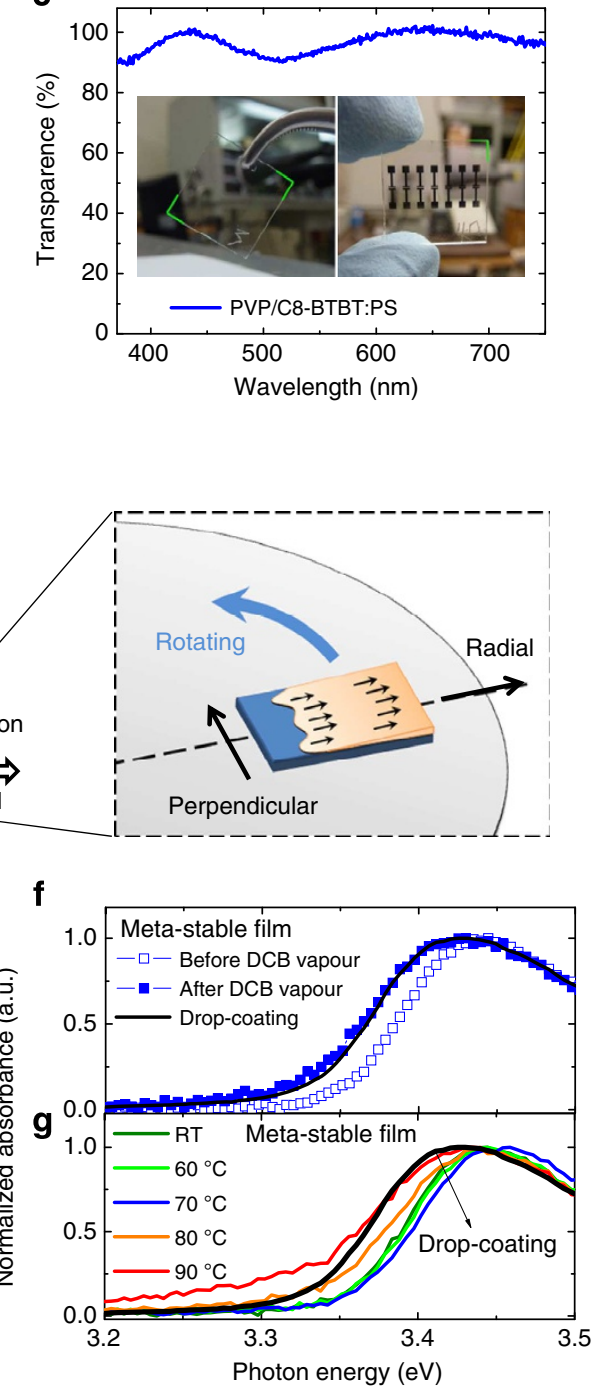

Figure 1 | Highly aligned meta-stable C8-BTBT:PS film fabricated by OCSC method. (a) Schematic device configuration of OTFT with C8-BTBT:PS blends as channel layer, PVP:HDA as dielectric layer and ITO as the gate electrode; the chemical structures of C8-BTBT, PS, PVP and HDA are shown. (b) Schematic illustration of the OCSC process, in which the substrates are located away from the axis of the spin-coater. (c) Transmission spectrum of the PVP:HDA/C8-BTBT:PS film. Inset: photographs of C8-BTBT:PS film and corresponding OTFT device with a structure of glass/ITO/PVP:HDA/C8-BTBT:PS. (d) Normalized polarized-absorption spectrum of OCSC C8-BTBT:PS film, where the light electrical field is in radial (blue circles) or perpendicular direction (red circles). The two directions are marked in the Fig. 1b; for comparison, the absorption peak of C8-BTBT film prepared by small-angle drop-coating, according to literature procedures ${ }^{25}$, is also shown ( $\mathbf{d}, \mathbf{f}, \mathbf{g}$, dark line). (e) Normalized polarized-absorption spectrum of on-centre spin-coated C8-BTBT:PS film, where the light electrical field is in radial (green circles) or perpendicular direction (pink circles). (f) Perpendicular polarized-absorption spectrum of meta-stable C8-BTBT:PS film before and after DCB vapour annealing. (g) Perpendicular polarized-absorption spectrum of meta-stable C8-BTBT:PS film after thermal annealing for $3 \mathrm{~h}$ at each temperature.

Since sample degradation currently precludes the full solution of the unit cell and molecular packing, we additionally characterized our highly aligned films using Near-edge X-ray Absorption Fine-Structure (NEXAFS) Spectroscopy. Figure 3 shows NEXAFS spectra for C8-BTBT films where the incidence angle of the highly polarized synchrotron X-rays was varied relative to the (100) direction (defined as orthogonal, Fig. 3a and Supplementary Fig. 8) and the (010) direction (defined as parallel, Fig. 3b). Similar results were obtained from C8-BTBT:PS films (Supplementary Fig. 9). The peaks near $285 \mathrm{eV}$ correspond to transition from the C1s core-level to antibonding $\pi^{*}$ orbitals of the conjugated backbone, where the intensity depends on the angle between the electric field vector and the direction of the final state $\left(\pi^{*}\right)$ orbitals ${ }^{32}$. The data show significant incident angular dependence and azimuthal anisotropy (difference between the two different sample orientations with respect to the beam). This shows a large degree of molecular order, an upright geometry (the $\pi^{*}$ signal is strongest at $90^{\circ}$ ), and an overall in-plane crystal alignment (the $\pi^{\star}$ signal is strongest with electric field vector along radial direction). The observed high in-plane alignment in NEXAFS is consistent with the anisotropic optical absorption spectra (Fig. 1d) and GIXD images (Supplementary Fig. 4). In Fig. 3c, the integrated $\pi^{*}$ intensity from 283.0 to $286.3 \mathrm{eV}$ is plotted versus the incidence angle of the parallel and orthogonal condition, respectively. By comparison with simulations according to the formalism for anisotropic NEXAFS intensity for a $\pi^{\star}$ vector outlined by Stohr and Outka $^{32}$, a transition dipole moment (TDM) tilt angle (the angle between TDM and the normal direction of substrate plane, Supplementary Fig. S8) of about $88 \pm 3^{\circ}$ was obtained. 

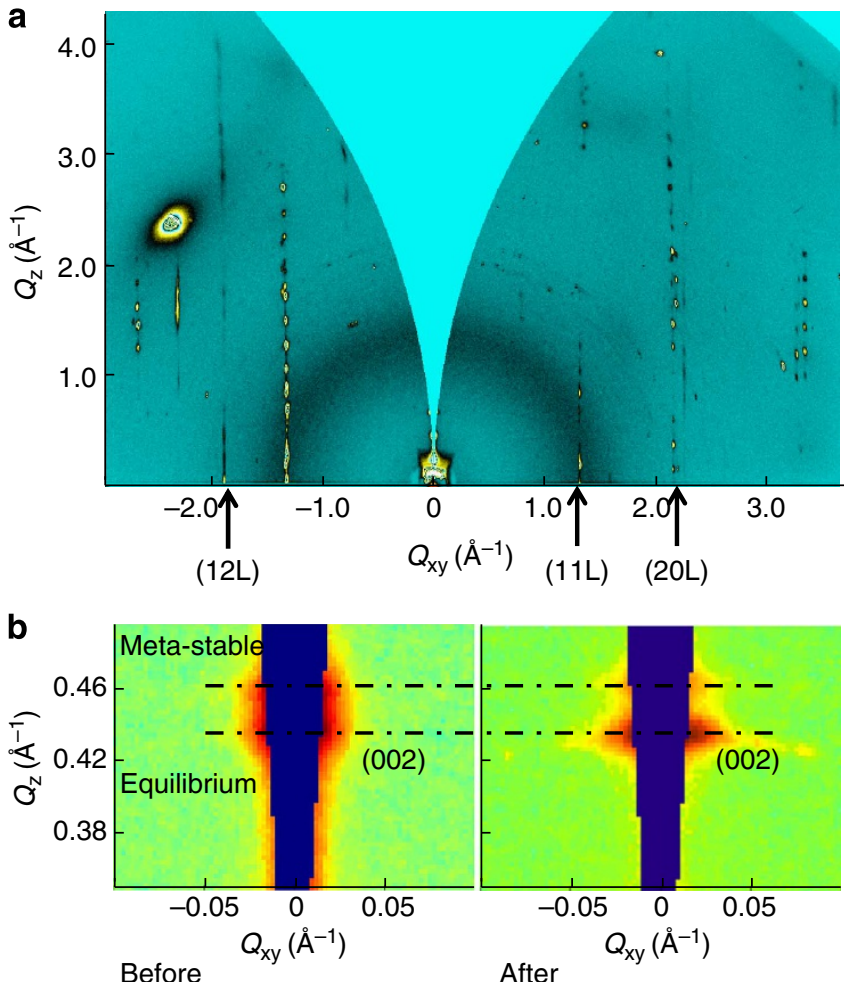

C

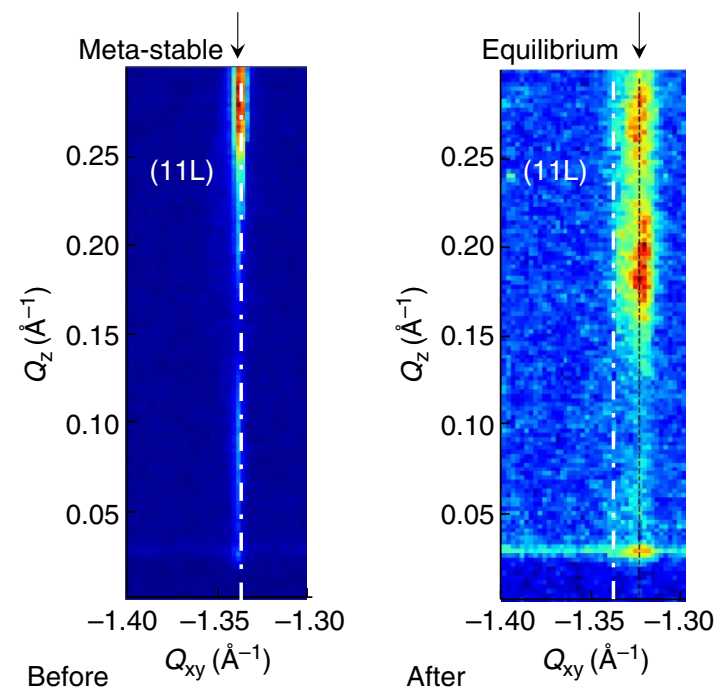

Figure 2 | GIXD investigations of highly aligned, meta-stable C8-BTBT

films. (a) GIXD images of an OCSC C8-BTBT samples. The presence of the asymmetric pattern is indicative of a high degree of in-plane alignment. The high order Bragg peaks indicate good crystallinity. There is an artifact due to the substrate at $Q_{x y}=-2.1 \AA^{-1}$ and $Q_{z}=2.1 \AA^{-1}$. (b) GIXD images of the (002) Bragg peaks of OCSC C8-BTBT:PS film obtained before and after thermal annealing, where the peak related to the meta-stable phase (top peak, $Q_{x y} \sim 0.46 \AA^{-1}$ ) is less intense compared with that from the equilibrium phase (bottom peak, $Q_{x y} \sim 0.44 \AA^{-1}$ ) after the thermal anneal. (c) GIXD images of the (11L) Bragg peaks of OCSC C8-BTBT:PS film obtained before and after thermal annealing. The in-plane position of (11L) Bragg peaks shifted after the film was heated to above $90^{\circ} \mathrm{C}$ and was subsequently cooled down to room temperature. The (11L) Bragg peaks first disappeared while the $(\mathrm{OOL})$ peaks remained visible, indicating the formation of a liquid crystalline phase (Supplementary Fig. 7b). The (11L) Bragg peaks then reformed as the film was cooled, but the (11L) peak shifted away from the original position, implying that the Bragg peak before heating was caused by a meta-stable phase.
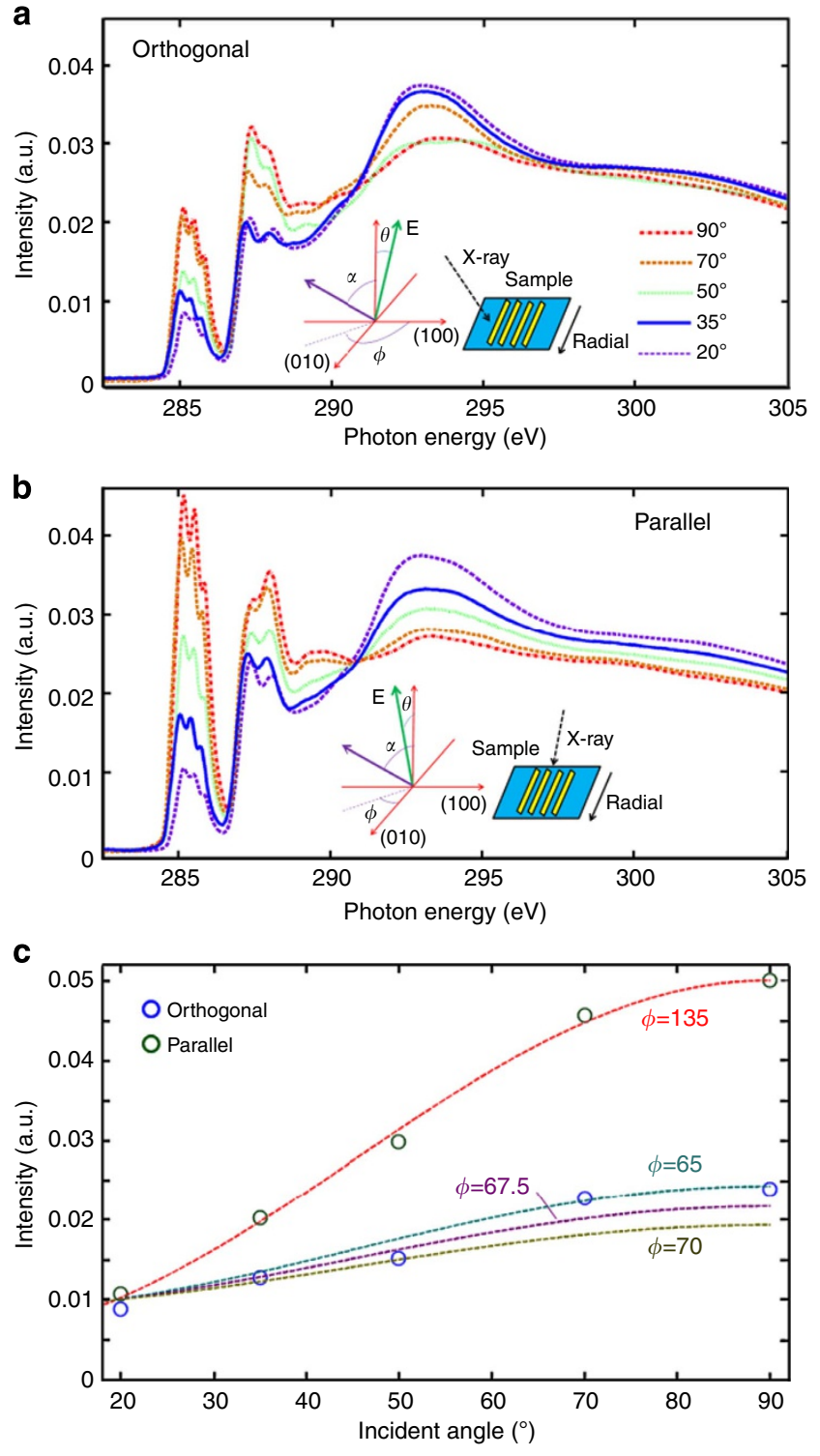

Figure 3 | NEXAFS investigations of C8-BTBT in-plane alignment.

(a) Carbon $\mathrm{K}$ edge NEXAFS spectra for the C8-BTBT:PS films at different $X$-ray incidence angles $(\theta$, i.e., the angle between the $X$-ray electric field $E$ and the normal direction of the substrate plane, where an incident angle of $90^{\circ}$ means the incident electric field is in the plane of the substrate. The orientation of the TDM is defined by the polar tilt angle $\alpha$ and the azimuthal angle $\phi$ ). The lower energy features centred around $285 \mathrm{eV}$ correspond to electronic transitions from core orbitals to antibonding $\pi^{\star}$ orbitals, whereas the higher energy feature centred around $293 \mathrm{eV}$ correspond to the transitions to unbound $\sigma^{\star}$ orbitals. Inset illustration shows the incident electric field of the polarized X-ray is orthogonal to the radial direction. (b) Corresponding NEXAFS spectra for the C8-BTBT:PS films with the incident electric field of the polarized $X$-ray being parallel to the radial direction. (c) Intensities of the $\pi^{\star}$ transitions versus incidence angle. $\pi^{\star}$ peaks were spectrally integrated from 283.0 to $286.3 \mathrm{eV}$, which is shown for both relative sample orientations (orthogonal and parallel) with respect to the incident polarization. The dash lines are the fitting curves with different azimuthal angle $\phi$. 
a

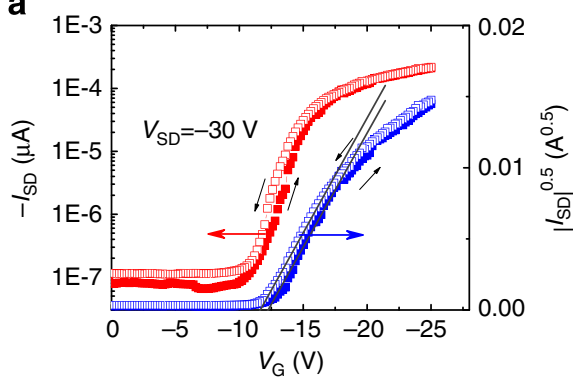

C

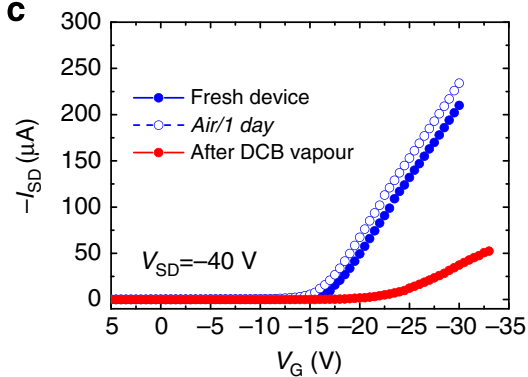

e

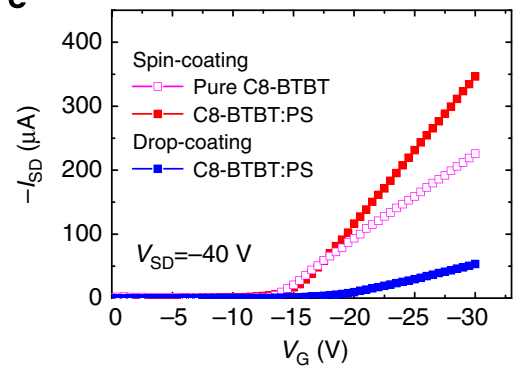

b

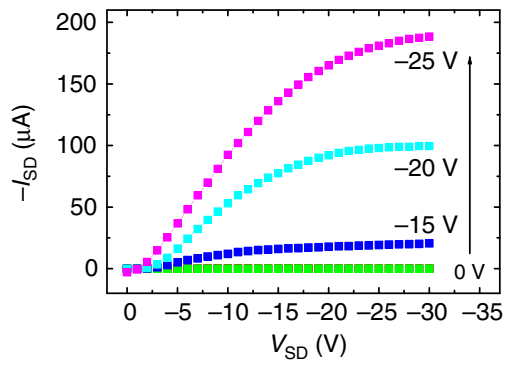

d
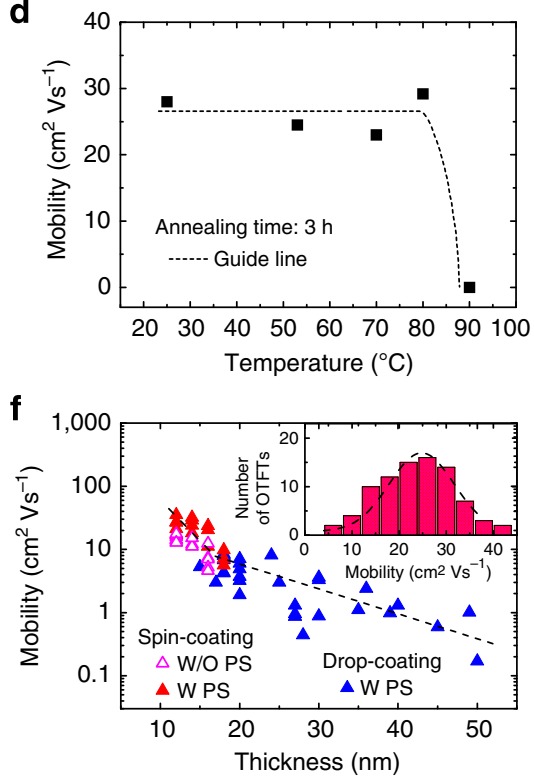

Figure 4 | High-performance OTFT devices with OCSC C8-BTBT:PS films. (a) Transfer and (b) Output characteristics of an OTFT prepared by OCSC with C8-BTBT:PS film as a channel layer. The channel length is $100 \mu \mathrm{m}$, channel width is $1 \mathrm{~mm}$ and the capacitance of the dielectric is $1.2 \times 10^{-4} \mathrm{Fm}{ }^{-2}$. (c) DCB vapour annealing for $2.5 \mathrm{~h}$ decreases the channel current. The channel current for the device kept in air for 1 day is also shown, excluding the possible explanation of device instability. (d) Hole carrier mobilities after thermal annealing for $3 \mathrm{~h}$ at each temperature. (e) Comparison of channel current of OTFT with OCSC channel layer or large-angle drop-coated channel layer. (f) Influence of the channel layer thickness and PS blending on the hole mobility. Inset: mobility distribution of the OCSC-prepared OTFTs.

(Supplementary Figs 9,10 and Supplementary Note 3). This is larger than that of the on-centre spun samples, which gave a TDM tilt angle of $77 \sim 81^{\circ}$ (Supplementary Fig. 11), suggesting a different molecular packing structure between off-centre spun films and conventionally on-centre spun films.

Hole mobilities in the OCSC C8-BTBT films. We investigated the hole transport characteristics of the OTFTs made with OCSC films (Fig. 4). These films showed an extremely high maximum hole mobility of $43 \mathrm{~cm}^{2} \mathrm{Vs}^{-1}$ for saturation mobility and $20 \mathrm{~cm}^{2} \mathrm{Vs}^{-1}$ for linear mobility. The average saturation mobility is $25 \mathrm{~cm}^{2} \mathrm{Vs}^{-1}$ (in set of Fig. $4 \mathrm{f}$ ). It should be noted that strikingly high saturation mobilities of $90 \sim 118 \mathrm{~cm}^{2} \mathrm{Vs}^{-1}$ were observed several times in some samples and the transfer current curves were shown in Supplementary Fig. 13. However, due to the lack of reproducibility, we report the maximum of $43 \mathrm{~cm}^{2} \mathrm{Vs}-1$ here as we observed similar value $\left(35 \sim 43 \mathrm{~cm}^{2} \mathrm{Vs}{ }^{-1}\right)$ in around $10 \%$ of more than 80 devices fabricated. These mobilities are the highest reported values for small-molecular organic semiconductors to date ${ }^{18,19,33-35}$. The high channel current of these devices has been independently verified by three research laboratories (Supplementary Fig. 14).

\section{Discussion}

The higher mobilities we obtained here is unlikely to be only attributed to a larger grain size as compared with previous studies, where single crystals were used in the channels ${ }^{18,24,25}$. Several additional factors may be contributing to the extremely high mobility: the highly aligned crystalline thin film, more continuous formation of C8-BTBT film due to the presence of the PS layer, passivation effect of the PS and possibly the meta-stable packing structure. While our films are highly aligned (Fig. 3 and Supplementary Figs 4,15), the higher mobility values do not originate from the intrinsic anisotropic change transport in the C8-BTBT crystal. This is because the mobilities along the radial and perpendicular directions were measured to be the same in absence of visible grain boundaries or cracks (Supplementary Fig. 16). The observed isotropic mobility is reasonable because the charge transfer integrals along different directions are roughly balanced $^{16,24}$. However, the high degree of alignment will still be a contributing factor to the high mobility value because such an alignment reduces the grain boundary scattering ${ }^{30}$.

The hole mobility of C8-BTBT has a strong dependence on the film thickness. Two coating methods, OCSC and large-angle drop-coating, were used to tune the C8-BTBT thickness within a range of $10-50 \mathrm{~nm}$. The OCSC films have typical thicknesses 
from 10 to $18 \mathrm{~nm}$, while the large-angle drop-coating films are around $15-50 \mathrm{~nm}$. In contrast to the formation of the thick C8BTBT crystals film in Figs 1b, a much larger tilting angle was used in the large-angle drop-coating process to reduce the C8-BTBT thickness to be $15-50 \mathrm{~nm}$ (Supplementary Methods). Independent of the two coating method, thinner films resulted in higher mobilities (Fig. 4e,f) in both cases, while the OCSC films showed the highest mobilities.

Second, we observed that blending PS into C8-BTBT yielded significantly higher mobilities (Fig. 4e,f and Supplementary Fig. 17). The higher mobility with PS may be due to enhanced inter-grain connectivity, because the increased solution viscosity helped to form more continuous films, as has been observed with other small molecule/polymer blends ${ }^{36,37}$. Another important contributing factor is the vertical phase separation, which generally occurs in polymer and small molecule blends ${ }^{36,38}$ and would result in reduced surface traps in our devices. To verify the vertical phase separation between C8-BTBT and PS, we studied the films with transmission electron microscopy (TEM) crosssectional images, where the samples were prepared by being embedded in epoxy resin and microtomed into 70-nm-thick slices. In conventional (unfiltered) cross-sectional TEM view (Fig. 5a), the C8-BTBT film is visible as a dark thin layer, due to its high crystallinity and electron density. An additional thinner layer $(\leq 5 \mathrm{~nm})$ is visible in places between the C8-BTBT layer and
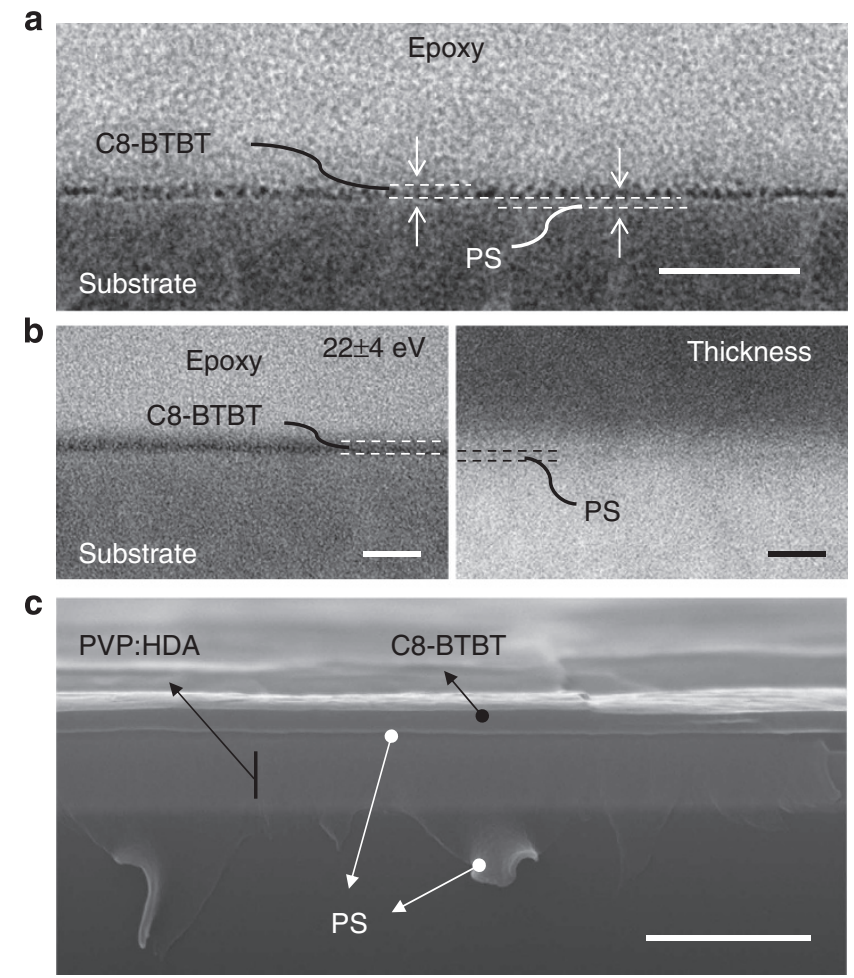

Figure 5 | Vertical phase separation between C8-BTBT and PS. (a) Crosssectional TEM of C8-BTBT:PS film without energy filtering. In an unfiltered cross-sectional TEM view, C8-BTBT film is visible as a dark thin layer with $10-20 \mathrm{~nm}$ thickness, due to its high crystallinity and electron density. The scale bar is $100 \mathrm{~nm}$. (b) Cross-sectional TEM of C8-BTBT:PS film with energy filtering. Both $22 \pm 4 \mathrm{eV}$ image and thickness map suggest that there is an additional layer underneath C8-BTBT. The two scale bars are $50 \mathrm{~nm}$. (c) Cross-sectional scanning electron microscopy (SEM) image of thick C8-BTBT:PS film drop coated on PVP surface, where the PS is more flexible than the C8-BTBT crystal and extended out of the cross section, demonstrating the PS segregation is energetically more favourable than the C8-BTBT segregation to the PVP surface. The scale bar is $1 \mu \mathrm{m}$. the substrate, which we attribute to the PS layer. We further confirmed the existence of this ultrathin PS layer by using energyfiltered TEM (Fig. 5b). The $22 \pm 4 \mathrm{eV}$ image utilized the plasmon peak of the p-type organic semiconductor to enhance the contrast in the electron energy loss spectra, and the thickness map is obtained from the ratio of unfiltered and filtered image, yielding pixel by pixel values of $t / \lambda$, where $t$ is film thickness in nanometre and $\lambda$ is the electron mean free pathway. Both the $22 \pm 4 \mathrm{eV}$ image and the thickness map suggest that there is indeed an additional layer underneath C8-BTBT ${ }^{39,40}$. Further indication of vertical phase separation in the C8-BTBT:PS film was observed by scanning electron microscopy using a thicker film with a higher percentage of PS, showing a similar phase-separated structure, where the PS layer was sandwiched between the C8-BTBT and PVP film (Fig. 5c). The vertical phase separation between C8-BTBT and PS is explained by their different surface energies. The PS segregation to the PVP surface is energetically more favourable than the segregation of C8-BTBT to the PVP surface, because the methyl-terminated C8-BTBT has lower surface energy than either PVP or PS (Supplementary Note 4).

Covering the PVP surface with an ultrathin layer of PS could reduce the interface traps caused by the polar hydroxyl groups on the PVP chain, contributing to the observed higher mobility ${ }^{41,42}$. Previously, the higher mobility in small molecule/polymer blends was also attributed to the 'zone refinement effect' during the vertical phase separation process, and this purification effect may also be present in this case ${ }^{43,44}$. Furthermore, the lower dielectric constant of PS $\left(\varepsilon_{\mathrm{r}}=2.6\right)$ than $\mathrm{PVP}\left(\varepsilon_{\mathrm{r}}=4.2\right)$ provides a less polar environment at the dielectric/C8-BTBT interface, leading to a less energetic disorder in the dielectric ${ }^{45}$. Both effects are expected to result in a higher OTFT mobility.

Finally, the meta-stable molecular packing in the OCSC film is also a contributing factor to the high mobility as can be seen from the much higher mobility of OCSC film compared with the similarly highly aligned drop-cast film (Supplementary Table 1). The presence of meta-stable packing was clearly supported by the peak shift in GIXD and the relaxation of the peak and shifting of the absorption spectrum to the equilibrium phase features. However, its contribution was difficult to quantify. For example, solvent annealing caused relaxation of the meta-stable polymorph (Figs 1f,g and 2c) accompanied by a hole mobility drop from $\sim 26 \mathrm{~cm}^{2} \mathrm{Vs}^{-1}$ to below $4.1 \mathrm{~cm}^{2} \mathrm{Vs}^{-1}$ (Fig. $4 \mathrm{c}, \mathrm{d}$ ). However, the crystal alignment was also disrupted (Supplementary Fig. 7), making it difficult to attribute the mobility drop entirely to crystal structure change. Moreover, the beam damage during GIXD measurements made it impossible to obtain the precise crystal packing structure for the meta-stable polymorph. Therefore, we were not able to determine the impact of the meta-stable packing on transfer integral.

It is not surprising that the ultra-high mobility achieved in the OCSC films combines the contributions from multiple effects. At current stage, it is difficult to pin-point the contribution of each factor quantitatively.

Given that we measured very high hole carrier mobilities in TFTs with highly aligned meta-stable C8-BTBT films, it is important to verify that the devices are robust with respect to bias stress. The performance of OTFTs fabricated with meta-stable C8-BTBT film was found to be quite stable under DC bias at room temperature. A source-drain current of $100 \mu \mathrm{A}$ can be maintained under a DC gate bias of $-15 \mathrm{~V}$ for over $1,000 \mathrm{~s}$ (Fig. 6), which indicates that the meta-stable C8-BTBT films did not undergo a relaxation to the equilibrium phase during continuous current flow. In addition, the low bias stress effect also indicates a negligible trap density in meta-stable C8-BTBT films, which can be attributed to the highly crystalline nature of the film and the presence of few grain boundaries as well as the 

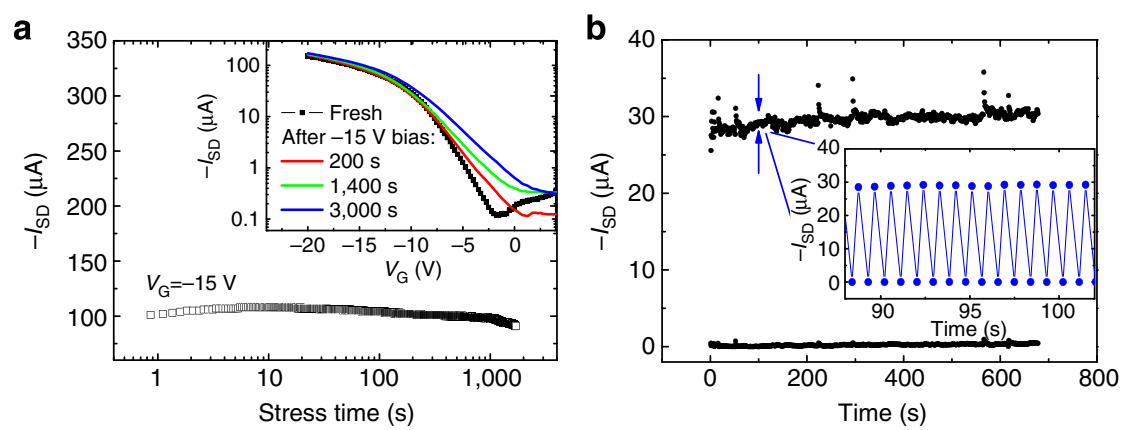

Figure 6 | Bias stress stability of the high mobility C8-BTBT:PS devices. (a) Stability of the channel current under a continuous bias stress of $-15 \mathrm{~V}$ for over $1,000 \mathrm{~s}$, inset show the $I_{S D}-V_{G}$ curve taken before and after bias stress. (b) Cycle stability of the device, where a train of gate voltage pulse ( $-10 \mathrm{~V}$ ) was applied and the device was switched between on and off for $\sim 750$ cycles $(1 \mathrm{~Hz})$. Inset show the detail $I_{S D}$ response at the 90 th seconds.

interfacial trap passivation by the ultrathin PS layer ${ }^{41,42,46,47}$. After a bias stress of over $200 \mathrm{~s}$, the threshold voltage started to decrease (Fig. 6a, inset). The origin of this shift is not yet fully understood; we hypothesize that this could be related to the motion of ions in the organic layer or the adsorption of moisture at the crosslinked PVP/C8-BTBT interface ${ }^{48,49}$. But the $I_{\mathrm{SD}}$ difference caused by the continuous bias becomes negligible when the $I_{\mathrm{SD}}$ exceeds $10 \mu \mathrm{A}$. The transistor also showed good cycle stability under repeated gate voltage pulses $(\sim 1 \mathrm{~Hz})$. The $I_{\mathrm{SD}}$ remains constant after 750 cycles of switching (Fig. 6b). Podzorov and coworkers ${ }^{50}$ have shown that the measured hole mobility of C8-BTBT is highly dependent on the gate voltage sweep rate: slowing down the gate voltage sweep rate decreased the mobility from $1 \mathrm{~cm}^{2} \mathrm{Vs}^{-1}$ to $0.05 \mathrm{~cm}^{2} \mathrm{Vs}^{-1}$. This sweep rate-dependent mobility results from the presence of trap states, which cause a strong concomitant stress bias effect. In this study, the device showed a negligible trap state density and thus robust stress bias stability, and no obvious sweep rate-dependent mobility was observed (Supplementary Fig. 18).

In summary, we have demonstrated a record high hole mobility of $43 \mathrm{~cm}^{2} \mathrm{Vs}^{-1}$ in OCSC C8-BTBT:PS films (the highest value obtained for small-molecular organic semiconductors) with transparency $>90 \%$ in the visible range. The OCSC films were considerably thinner than what previously studied and have meta-stable structure. These meta-stable films were observed to maintain its structural integrity up to $80^{\circ} \mathrm{C}$, and the subsequent fabricated devices were stable under both DC and AC bias at room temperature. Our data indicate that the obtained very high hole mobility in C8-BTBT:PS blend films mainly results from the highly aligned crystalline grains with a slightly reduced in-plane intermolecular spacing. The obtained mobility also clearly benefited from PS blending via the formation of vertical phase separation, where the PS segregated to the dielectric/semiconductor interface may have helped to reduce interfacial traps. Collectively, this study demonstrates a new method to enhance the performance of OTFTs. The highest obtained hole mobility is comparable to that of the polycrystalline silicon, indicating a bright future for OTFT applications.

\footnotetext{
Methods

Device fabrication. For the device fabrication, either ITO-coated glass substrates or highly doped silicon wafers were used. The substrates were first scrubbed with a brush dipped in acetone and ultrasoniced in pure water, acetone and isopropyl alcohol, respectively. Then these substrates were dried in oven at $80^{\circ} \mathrm{C}$. After UV-ozone treatment, the ITO layer was covered by a low temperature cross-linkable PVP dielectric layer according to literature procedures ${ }^{27}$. Here $4,4^{\prime}$-(hexafluoroisopropylidene)diphthalic anhydride (HDA) was used as the cross linker. The PVP-HDA layer was spin-coated from a $100 \mathrm{mg} \mathrm{ml}^{-1}$ solution of PVP:HDA (10:1 by wt) in propylene glycol monomethyl ether acetate. The PVP:HDA films were cured at $100^{\circ} \mathrm{C}$ for $60 \mathrm{~min}$ to promote the cross-linking
}

reaction, resulting in a dielectric layer thickness of $\sim 330 \mathrm{~nm}$ and a measured capacitance of $1.2 \times 10^{-4} \mathrm{~F} \mathrm{~m}^{-2}$ (Supplementary Fig. 1).

The organic semiconductor films were deposited in an nitrogen inert atmosphere on PVP-HDA-coated ITO substrate from a $5 \mathrm{mg} \mathrm{ml}^{-1} \mathrm{C} 8$-BTBT (or C8-BTBT:PS) solution in DCB via a OCSC method (Supplementary Fig. 2a), where the substrate $\left(15 \times 15 \mathrm{~mm}^{2}\right)$ was placed with its centre away from the rotation axis of the spin-coater at a distance of $20-40 \mathrm{~mm}$. During the OCSC process, the spin speed was gradually increased to 2,700 r.p.m. (Supplementary Fig. 2b). The C8-BTBT crystals grow gradually from one side to the other side in the radial direction. The sample was brought out of the $\mathrm{N}_{2}$-golve box immediately after spincoating. The C8-BTBT:PS semiconductor layer fabricated on the PVP surface had an thickness of $10-18 \mathrm{~nm}$, which was varied by changing both spin-coating speed and the solution concentration. Finally, silver (Ag) source and drain electrodes were thermally evaporated through a Si shadow mask with a channel length of $100 \mu \mathrm{m}$ and a channel width of $1 \mathrm{~mm}$, respectively. The electrical characteristics of the devices were measured with two computer-controlled Keithley 2400 source metre in ambient conditions.

Polarized absorption spectroscopy measurement. For the polarized absorption spectrum measurements, the C8-BTBT (or C8-BTBT:PS) films were spin coated on PVP:HDA covered quartz with a size of about $25 \times 25 \mathrm{~mm}^{2}$. Absorption was measured with a UV-Visible spectrophotometer (Thermo Scientific, Evolution 201) combined with a polarizer. The size of the light spot was about $1 \times 3 \mathrm{~mm}^{2}$. The stability of the meta-stable C8-BTBT:PS film was characterized by optical absorption spectroscopy with the polarization of light in the perpendicular direction (Supplementary Fig. 3). The absorption spectrum of the meta-stable C8-BTBT:PS film was collected multiple times over the course of 1 month storage, during which the C8-BTBT:PS film was kept in the dark at ambient conditions. All the optical measurements were carried out on the same region of the film, with a positional error of about $1 \sim 2 \mathrm{~mm}$.

GIXD measurement. GIXD was performed at the Stanford Synchrotron Radiation Lightsource (SSRL) on beamlines 11-3 and 7-2. The photon energy was $12.73 \mathrm{keV}$ in beamline 11-3 and $8 \mathrm{keV}$ in beamline 7-2. The scattering intensity was recorded on a $2 \mathrm{D}$ image plate (MAR-345) with a pixel size of $150 \mu \mathrm{m}(2,300 \times 2,300$ pixels), located at a distance of either $150 \mathrm{~mm}$ or $400 \mathrm{~mm}$ from the sample centre for beamline 11-3, and with a point detector for beamline 7-2. The distance between the sample and the detector was calibrated using lanthanum hexaboride $\left(\mathrm{LaB}_{6}\right)$ polycrystalline standard (beamline 11-3). The incidence angle was chosen in the range of $0.10^{\circ}-0.14^{\circ}$. The beam size was $50 \times 150 \mu \mathrm{m}$ (vertically and horizontally), which resulted in a $150 \mu \mathrm{m}$ wide beam footprint on the sample that spanned the length of the $2 \sim 3 \mathrm{~mm}$ sample. The data were distortion-corrected (theta-dependent image distortion introduced by planar detector surface) before performing quantitative analysis on the images. Numerical integration of the diffraction peak areas was performed with the software $\mathrm{WxDiff}^{51}$. The overall resolution in the GIXD experiments, largely determined by the sample size $(2-3 \mathrm{~mm})$, was about $0.01 \AA^{-1}$.

NEXAFS measurement. NEXAFS measurements were performed at the bending magnet beamline 8-2 of SSRL ${ }^{52}$, with a ring current of $500 \mathrm{~mA}$, operating the spherical grating monochromator with the $500 \mathrm{lmm}^{-1}$ grating at intermediate $(\sim 0.3 \mathrm{eV})$ resolution. The toroidal refocusing optics provided a near circular beam cross-section of about $1 \mathrm{~mm}$ FWHM (full width at half maximum) in diameter (footprint horizontally was 1-3 mm FWHM pending on the incidence angle). All the samples were mounted in a single load to an aluminium sample holder using conductive carbon, and all the measurements were performed at room temperature and under ultra-high vacuum conditions (below $10^{-8}$ Torr). Both the total electron yield and the Auger electron yield were recorded by means of the sample 
drain current (measured via a stanford research systems (SRS) current amplifier without bias) and a $\phi 15-255 \mathrm{G}$ double-pass cylindrical mirror analyser operated in pulse counting mode and at a fixed kinetic energy of $257 \mathrm{eV}$ at $200 \mathrm{eV}$ pass energy. Total electron yield was chosen for the analysis in this work. After dark current subtraction, the sample current was normalized to the incoming photon flux, recorded from a freshly Au-evaporated gold mesh that intercepts 20\% of the beam upstream of the chamber. A linear pre-edge background signal was then subtracted, and the spectra were normalized to the total area. The polarization factor $\mathrm{P}$ (defined as the ratio of the in-plane component to the total intensity) of the elliptically polarized synchrotron radiation was assumed to be $90 \%$ (ref. 32).

TEM Measurement. For cross-section TEM experiments, the sample films were embedded in low viscosity epoxy and microtomed into thin slices with a thickness of 50-100 nm. TEM experiments were performed in a Zeiss Libra 120 which is equipped with an in-column energy filter. An acceleration voltage of $120 \mathrm{kV}$ was used along with an emission current as small as $5 \times 10^{-6} \mathrm{~A}$ and a minimal beam intensity to avoid electron beam induced morphological change. To examine the nanomorphology of C8-BTBT:PS films across the thickness direction, cross-sectional TEM samples were prepared by embedding the sample films in epoxy resin and microtoming into 70 -nm-thick slices.

\section{References}

1. Rogers, J. A. \& Bao, Z. Printed plastic electronics and paperlike displays. J. Polym. Sci., Part A: Polym. Chem. 40, 3327-3334 (2002).

2. Gelinck, G. H. et al. Flexible active-matrix displays and shift registers based on solution-processed organic transistors. Nat. Mater. 3, 106-110 (2004).

3. Subramanian, V. et al. Progress toward development of all-printed RFID tags: materials, processes, and devices. Proc. IEEE 93, 1330-1338 (2005).

4. Baude, P. et al. Pentacene-based radio-frequency identification circuitry. Appl. Phys. Lett. 82, 3964-3966 (2003).

5. Klauk, H., Zschieschang, U., Pflaum, J. \& Halik, M. Ultralow-power organic complementary circuits. Nature 445, 745-748 (2007).

6. Sirringhaus, H. et al. High-resolution inkjet printing of all-polymer transistor circuits. Science 290, 2123-2126 (2000).

7. Crone, B. et al. Large-scale complementary integrated circuits based on organic transistors. Nature 403, 521-523 (2000).

8. Someya, T., Dodabalapur, A., Huang, J., See, K. C. \& Katz, H. E. Chemical and physical sensing by organic field-effect transistors and related devices. Adv. Mater. 22, 3799-3811 (2010).

9. Lipomi, D. J. et al. Skin-like pressure and strain sensors based on transparent elastic films of carbon nanotubes. Nat. Nanotech. 6, 788-792 (2011).

10. Mannsfeld, S. C. et al. Highly sensitive flexible pressure sensors with microstructured rubber dielectric layers. Nat. Mater. 9, 859-864 (2010).

11. Kuribara, K. et al. Organic transistors with high thermal stability for medical applications. Nat. Commun. 3, 723 (2012).

12. Roberts, M. E. et al. Water-stable organic transistors and their application in chemical and biological sensors. Proc. Natl Acad. Sci. USA 105, 12134-12139 (2008).

13. Berggren, M. \& Richter-Dahlfors, A. Organic bioelectronics. Adv. Mater. 19, 3201-3213 (2007)

14. Brédas, J.-L., Beljonne, D., Coropceanu, V. \& Cornil, J. Charge-transfer and energy-transfer processes in $\pi$-conjugated oligomers and polymers: a molecular picture. Chem. Rev. 104, 4971-5004 (2004).

15. Sokolov, A. N. et al. From computational discovery to experimental characterization of a high hole mobility organic crystal. Nat. Commun. 2, 437 (2011).

16. Takimiya, K., Shinamura, S., Osaka, I. \& Miyazaki, E. Thienoacene-based organic semiconductors. Adv. Mater. 23, 4347-4370 (2011).

17. Yuan, Y. et al. Solution-processed nanoparticle super-float-gated organic fieldeffect transistor as un-cooled ultraviolet and infrared photon counter. Sci. Rep. 3, 2707 (2013).

18. Minemawari, H. et al. Inkjet printing of single-crystal films. Nature 475, 364-367 (2011)

19. Giri, G. et al. Tuning charge transport in solution-sheared organic semiconductors using lattice strain. Nature 480, 504-508 (2011).

20. Diao, Y. et al. Solution coating of large-area organic semiconductor thin films with aligned single-crystalline domains. Nat. Mater. 12, 665-671 (2013).

21. Ruiz, R. et al. Structure of pentacene thin films. Appl. Phys. Lett. 85, 4926-4928 (2004)

22. Gundlach, D., Jackson, T., Schlom, D. \& Nelson, S. Solvent-induced phase transition in thermally evaporated pentacene films. Appl. Phys. Lett. 74, 3302-3304 (1999).

23. Yoshida, H., Inaba, K. \& Sato, N. X-ray diffraction reciprocal space mapping study of the thin film phase of pentacene. Appl. Phys. Lett. 90, 181930 (2007).

24. Soeda, J. et al. Solution-crystallized organic field-effect transistors with charge-acceptor layers: high-mobility and low-threshold-voltage operation in air. Adv. Mater. 23, 3309-3314 (2011)
25. Liu, C. et al. Solution-processable organic single crystals with bandlike transport in field-effect transistors. Adv. Mater. 23, 523-526 (2011).

26. Uemura, T., Hirose, Y., Uno, M., Takimiya, K. \& Takeya, J. Very high mobility in solution-processed organic thin-film transistors of highly ordered [1] benzothieno [3, 2-b] benzothiophene derivatives. Appl. Phys. Express 2, 111501-111503 (2009).

27. Roberts, M. E. et al. Cross-linked polymer gate dielectric films for low-voltage organic transistors. Chem. Mater. 21, 2292-2299 (2009).

28. Nomura, K. et al. Room-temperature fabrication of transparent flexible thinfilm transistors using amorphous oxide semiconductors. Nature 432, 488-492 (2004).

29. Park, S. H. K. et al. Transparent and photo-stable zno thin-film transistors to drive an active matrix organic-light-emitting-diode display panel. Adv. Mater 21, 678-682 (2009).

30. Rivnay, J. et al. Large modulation of carrier transport by grain-boundary molecular packing and microstructure in organic thin films. Nat. Mater. 8, 952-958 (2009).

31. Izawa, T., Miyazaki, E. \& Takimiya, K. Molecular ordering of high-performance soluble molecular semiconductors and re-evaluation of their field-effect transistor characteristics. Adv. Mater. 20, 3388-3392 (2008).

32. Stöhr, J. \& Outka, D. Determination of molecular orientations on surfaces from the angular dependence of near-edge $\mathrm{x}$-ray-absorption fine-structure spectra. Phys. Rev. B 36, 7891 (1987).

33. Takeya, J. et al. Very high-mobility organic single-crystal transistors with in-crystal conduction channels. Appl. Phys. Lett. 90, 102120 (2007).

34. Jurchescu, O. D., Baas, J. \& Palstra, T. Effect of impurities on the mobility of single crystal pentacene. Appl. Phys. Lett. 84, 3061-3063 (2004).

35. Li, H. et al. High-mobility field-effect transistors from large-area solutiongrown aligned c60 single crystals. J. Am. Chem. Soc. 134, 2760-2765 (2012).

36. Smith, J. et al. Solution-processed organic transistors based on semiconducting blends. J. Mater. Chem. 20, 2562-2574 (2010).

37. Kang, J., Shin, N., Jang, D. Y., Prabhu, V. M. \& Yoon, D. Y. Structure and properties of small molecule - polymer blend semiconductors for organic thin film transistors. J. Am. Chem. Soc. 130, 12273-12275 (2008).

38. Hamilton, R. et al. High-performance polymer-small molecule blend organic transistors. Adv. Mater. 21, 1166-1171 (2009).

39. Chen, J. et al. Ternary behavior and systematic nanoscale manipulation of domain structures in P3HT/PCBM/P3HT-b-PEO films. J. Mater. Chem. 22, 13013-13022 (2012).

40. Drummy, L. F. et al. Molecular-scale and nanoscale morphology of P3HT: PCBM bulk heterojunctions: energy-filtered TEM and low-dose HREM. Chem. Mater. 23, 907-912 (2010).

41. Baeg, K. J., Noh, Y. Y., Sirringhaus, H. \& Kim, D. Y. Controllable shifts in threshold voltage of top-gate polymer field-effect transistors for applications in organic nano floating gate memory. Adv. Funct. Mater. 20, 224-230 (2010).

42. Park, B. et al. Enhanced hole mobility in ambipolar rubrene thin film transistors on polystyrene. Appl. Phys. Lett. 92, 133302 (2008).

43. Chung, Y. S. et al. Zone-refinement effect in small molecule - polymer blend semiconductors for organic thin-film transistors. J. Am. Chem. Soc. 133, 412-415 (2010).

44. Li, Y. et al. In situ purification to eliminate the influence of impurities in solution-processed organic crystals for transistor arrays. J. Mater. Chem. C 1, 1352-1358 (2013).

45. Sirringhaus, H. Device physics of solution-processed organic field-effect transistors. Adv. Mater. 17, 2411-2425 (2005).

46. Klauk, H. Organic Electronics: Materials, Manufacturing, and Applications (Wiley-VCH, 2006).

47. Chang, J. B. \& Subramanian, V. Effect of active layer thickness on bias stress effect in pentacene thin-film transistors. Appl. Phys. Lett. 88, 233513 (2006).

48. Kumaki, D., Umeda, T. \& Tokito, S. Influence of HO and O on threshold voltage shift in organic thin-film transistors: deprotonation of $\mathrm{SiOH}$ on $\mathrm{SiO}$ gate-insulator surface. Appl. Phys. Lett. 92, 093309 (2008).

49. Kim, C. S., Lee, S., Gomez, E. D., Anthony, J. E. \& Loo, Y.-L. Solvent-dependent electrical characteristics and stability of organic thin-film transistors with drop cast bis (triisopropylsilylethynyl) pentacene. Appl. Phys. Lett. 93, 103302 (2008).

50. Chen, Y. et al. Dynamic character of charge transport parameters in disordered organic semiconductor field-effect transistors. Phys. Chem. Chem. Phys. 14, 14142-14151 (2012).

51. Mannsfeld, S. C., Tang, M. L. \& Bao, Z. Thin film structure of triisopropylsilylethynyl-functionalized pentacene and tetraceno $[2,3-\mathrm{b}]$ thiophene from grazing incidence X-Ray diffraction. Adv. Mater. 23, 127-131 (2011).

52. Tirsell, K. G. \& Karpenko, V. P. A general purpose sub-kev x-ray facility at the stanford synchrotron radiation laboratory. Nucl. Instrum. Methods Phys. Res. Sect. A 291, 511-517 (1990). 


\section{Acknowledgements}

This work was financially supported by Defense Advanced Research Projects Agency under the award W31P4Q-08-C-0439 through Agiltron Inc. and the National Science Foundation (DMR-1303178, ECCS-1348272 and CMMI-1265834) and Air Force Office of Scientific Research (FA9550-12-1-0190). The HRTEM was conducted at the Center for Nanophase Materials Sciences, which is sponsored at Oak Ridge National Laboratory by the Scientific User Facilities Division, Office of Basic Energy Sciences, US Department of Energy. Portions of this research were carried out at the Stanford Synchrotron Radiation Lightsource, a national user facility operated by Stanford University on behalf of the US Department of Energy, Office of Basic Energy Sciences. We thank Dr Hylke B. Akkerman and Dr Gerwin H. Gelinck in Holst Centre for the verification of the transfer curves.

\section{Author contributions}

J.H. and Z.B. conceived the project and designed the experiments. Y.Y. designed the off-centre spin-coating method, carried out all the device fabrication and characterizations, the optical absorption measurement, SEM, and related data analysis. G.G. and S.C.B.M. carried out the GIXD measurements and molecular structure analysis. A.A. and
Y.Y. carried out the data analysis of the optical absorption measurements. A.L.A., D.N. and M.T. carried out the NEXAFS measurements and data analysis. J.C. carried out the TEM measurement. A.P.Z. synthesized the C8-BTBT material. All the authors analysed and interpreted the data and wrote the paper.

\section{Additional information}

Supplementary Information accompanies this paper at http://www.nature.com/ naturecommunications

Competing financial interests: The authors declare no competing financial interests.

Reprints and permission information is available online at http://npg.nature.com/ reprintsandpermissions/

How to cite this article: Yuan, Y. et al. Ultra-high mobility transparent organic thin film transistors grown by an off-centre spin-coating method. Nat. Commun. 5:3005 doi: $10.1038 /$ ncomms4005 (2014) 